\title{
Élise JULIEN, Paris, Berlin. La mémoire de la guerre
}

1914-1933

\section{Anthony Michel}

\section{OpenEdition}

Journals

Édition électronique

URL : http://journals.openedition.org/questionsdecommunication/301

DOI : 10.4000/questionsdecommunication.301

ISSN : 2259-8901

\section{Éditeur}

Presses universitaires de Lorraine

\section{Édition imprimée}

Date de publication : 1 décembre 2010

Pagination : 280-282

ISBN : 978-2-8143-0056-9

ISSN : 1633-5961

\section{Référence électronique}

Anthony Michel, «Élise JuLien, Paris, Berlin. La mémoire de la guerre 1914-1933 », Questions de communication [En ligne], 18 | 2010, mis en ligne le 09 mai 2012, consulté le 22 septembre 2020. URL: http://journals.openedition.org/questionsdecommunication/301 ; DOI : https://doi.org/10.4000/ questionsdecommunication.301 
Élise JULIEN, Paris, Berlin. La mémoire de la guerre 19/41933.

Rennes, Presses universitaires de Rennes, coll. Histoire, 2009, 375 p.

Cet ouvrage est une version remaniée de la thèse de doctorat de l'auteure qui pose comme problématique initiale la question suivante : peut-on discerner à Paris et à Berlin une mémoire spécifique de la guerre? Son étude part d'un constat simple: aucune des deux capitales «n'a érigé le monument qui aurait pu revenir à leurs morts » (p. 9). C'est ainsi qu'elle s'est intéressée à une comparaison de l'histoire de la mémoire de la Première Guerre mondiale, construite matériellement entre ces deux villes. Le livre est découpé en trois parties. La première, intitulée « France et Allemagne : les cadres nationaux de la mémoire de la guerre », pose un cadre sur la position historico-socio-politique des deux pays avant, pendant et après le conflit. Cela permet de comprendre comment chacun se situe visà-vis de la mémoire de ses morts et d'une manière plus générale de la commémoration, en fonction de la manière dont il a vécu le conflit et comment il en est sorti. La deuxième concerne « Paris et Berlin : la fragilité des mémoires urbaines globales ». Dans celle-ci, Élise Julien explique la construction, l'évolution et l'importance de la mémoire commune dans chaque ville, en rapport à sa « nationalisation ». Enfin, la dernière partie, « Paris et Berlin : la diversité des mémoires urbaines locales », présente une série d'études de cas de la construction mémorielle du conflit selon deux angles: les communautés institutionnelles et l'ancrage territorial.

Par ce sommaire, on peut déjà se rendre compte de la trame que prendra cette comparaison. À savoir trois thèmes spécifiques: le national, le communal et le très local. Chaque partie introduit une échelle différente dans laquelle plusieurs études de cas ont été réalisées, notamment sur les multiples communautés et la relation au territoire. Étant donnée la position des villes à l'arrière du front, on précise aussi que ce sont des mémoires urbaines, donc des mémoires qui divergent de celles rapportées par les combattants. De plus, avant de commencer son analyse comparative, l'auteure explique deux points : elle arrête son travail en 1933, car c'est au cours de cette année que les nazis prennent le pouvoir et instrumentalisent politiquement la mémoire de la guerre; donc, pour elle, il n'y a plus d'intérêt pour son étude; elle précise les contours de son corpus composé de publications officielles des politiques publiques, des archives des communautés privées et institutionnelles et de quelques titres de la presse généraliste à grand tirage.

L'auteure rappelle d'abord que « chaque mémoire collective est pensée comme une interaction entre expérience vécue ou transmise et élaborations institutionnelles, officielles ou historiques; elle demeure susceptible d'évoluer en permanence » (p. 12). Ainsi faut-il y voir une éventuelle manipulation - positive comme négative - des pouvoirs politiques selon une logique du présent. Comme le souligne Élise Julien, « leur vocation de centres de pouvoir (à Paris et Berlin) en fait des terrains privilégiés pour l'expression des politiques publiques ») (p. 18). Elle évoque alors la Résistance pour la capitale française et la mobilisation en faveur de l'effort de guerre pour la capitale allemande. Se pose également la question des silences et des oublis, d'où une mémoire collective évolutive. De ce fait, plusieurs questions se posent à l'égard de la commémoration : quel est son impact et son rôle dans le deuil des individus, comment qualifiet-elle l'expérience de guerre?

La première partie présente les cadres généraux de la mémoire de la guerre dans les deux pays. La mémoire collective, les commémorations et les hommages sont le fruit d'une négociation permanente entre l'influence des politiques publiques et les revendications de la société civile. La multiplication des pratiques commémoratives - manifestation visible de la mémoire - « reflète la profondeur du traumatisme causé par la guerre sur les individus » (p.74) mais elle console les endeuillés, rappelle l'héroïsme de ceux qu'ils pleurent et institue un exemple moral pour l'éducation des générations futures. Cependant, le contexte sociopolitique influence beaucoup la façon dont sont célébrés les morts. L'issue du conflit est une source de divergence entre les deux pays. En Allemagne, on tente de masquer la défaite qui n'est pas admise, alors qu'en France l'optimisme victorieux diminue progressivement. Si le deuil 
privé et le culte public sont présents sur les deux territoires, le sens de la mort est différent. En France, il y a de réels « symboles nationaux unificateurs 》, avec le II-Novembre comme fête nationale, alors qu'en Allemagne, il y a « différentes interprétations politiques» (p. |10), entrainant une pluralité de dates commémoratives. On comprend alors que la mémoire devient un enjeu politique potentiellement déstabilisateur. Les États cherchent donc « à s'en emparer et à l'intégrer dans un contexte mémoriel plus vaste qui puisse lui donner un sens utile à l'avenir national » (p. |46). Deux nouveaux points de vue s'opposent encore. Si l'État s'occupe de la commémoration nationale côté français, ce sont les associations privées qui s'en chargent côté allemand. Et c'est justement cette distinction que va développer l'auteure dans sa deuxième partie, analysant la spécificité de la mémoire de la guerre à l'échelle des deux villes.

Paris et Berlin ont vécu différemment la Grande Guerre. La première ville était le lieu du ravitaillement alors que la seconde, loin du front, n'a jamais été menacée militairement. Cependant, la mémoire de guerre dans ces capitales se construit par l'intermédiaire d'interventions d'acteurs nationaux composés de « groupements de l'État, religieux, politiques, corporatifs » (p. 185). Une idée est également partagée que pour rendre audible un discours, il faut en investir la capitale pour gagner une envergure nationale. Si l'Arc de Triomphe et la tombe du Soldat inconnu sont les monuments à Paris, un tel projet était prévu à Berlin mais les divergences à propos du symbole et de l'emplacement ne trouvant pas d'accord, rien ne fut fait. Que ce soit pour lesdits monuments parisiens ou ceux dispersés dans la capitale berlinoise, tous « accèdent au statut de monuments nationaux, contribuant ainsi à une forme de nationalisation de la mémoire monumentale » (p. 219), même si les premiers participent à un symbole national ce qui n'est pas le cas des seconds, dont la conscience nationale est hétérogène. Seule différence entre les deux villes, le choix de la commémoration. Contrairement à Berlin, Paris peut choisir entre célébrer la communauté victorieuse ou de deuil.

Cette divergence est perceptible grâce aux actions parallèles mais conjointes des institutions religieuses, comme associatives et professionnelles, qui érigent des monuments pour leurs membres. Elise Julien explique d'ailleurs que « ces monuments ont pour fonction interne de renforcer l'intégrité du groupe par la réactivation solennelle du souvenir de ses disparus » (p. 230). En revanche, les communautés politiques ont du mal à s'imposer. Et la politique mémorielle des villes est plutôt le résultat d'initiatives multiples prenant « le relais de l'interventionnisme municipal des années de guerre ». D'un point de vue territorial, le cimetière est l'exemple le plus significatif : lieu du recueillement, de la piété privée et du rapport intime à la mort, il devient plus souvent le centre de cérémonies d'hommage collectif. On renomme également les rues de noms de personnalités. La commune et la paroisse sont des pôles d'identité collective entre l'individu et la nation. Si des clivages s'opposent entre les partis politiques la gauche veut tourner la page et la droite dénonce le déni du passé - sur un monument national concret, les arrondissements parisiens en profitent pour élever leur propre monument. II en va de même à Berlin, avec la multiplication des monuments communaux entrainant la maitrise de l'espace public sur la capitale.

La troisième partie s'intéresse justement à la façon dont se jouent l'identité et la mémoire des Parisiens et Berlinois. La mémoire locale est le fruit des commémorations des communautés institutionnelles en lien avec un lieu ou un territoire. On retrouve ainsi des groupes scolaires, universitaires, professionnels, militaires et religieux. Chaque mémoire thématique a son monument aux morts. Cette partie du livre est composée d'une série d'exemples comparatifs dans chaque groupe cité ci-dessus. On n'en retiendra que quelques-uns. Chaque monument présenté dans cette partie fait l'objet d'une monographie illustrée. La commémoration scolaire de la guerre maintient le lien entre ceux partis au front et ceux restés à l'arrière. Le monument construit sous le préau est garant de la cohérence et de la continuité de la communauté scolaire. Cet aspect est illustré par la comparaison entre l'université de Berlin et l'École normale supérieure à Paris. La mémoire professionnelle est étudiée avec Siemens côté allemand et la Société du Gaz, côté français. Une même étude a également été réalisée par l'auteure à l'échelle d'arrondissements parisiens et de communes berlinoises. Chaque communauté est une « parentèle fictive », définie comme un groupe d'acteurs de la mémoire liés non par le sang mais par l'expérience, qui a besoin de s'exprimer et d'agir ensemble. De plus, ces communautés et donc ces mémoires sociales sont en interaction car elles se partagent des territoires. Ainsi les stratégies monumentales s'élaborent-elles à la fois dans le cadre d'enjeux de pouvoirs locaux et en rapport à un espace urbain concret, préexistant et en évolution. Ce que l'on retient principalement de ces exemples comparatifs est l'emprise du pouvoir 
mémoriel des arrondissements, des communes voire des communautés institutionnelles sur le politique à l'échelle des capitales.

En conclusion, par l'intermédiaire des nombreux exemples justifiés, juxtaposés - parisiens puis berlinois - et illustrés, et grâce au cheminement astucieusement choisi par Élise Julien - du national au très local -, on comprend que la construction mémorielle des capitales est difficile et passe par une mobilisation homogène d'institutions sociales multiples. Cela participe à la façon dont les habitants font ou non leur propre histoire. Chaque monument élevé s'accompagnant du triptyque « consoler conserver - interpréter » doit participer, comme le dit l'auteure, à ce que «l la mémoire historique se détache progressivement des mémoires vives et qu'elle s'oriente finalement vers la logique patrimoniale actuelle » (p. 375).

Anthony Michel

CREM, université Paul Verlaine-Metz michelanthony@yahoo.fr 